\title{
FINITE-TIME RUIN PROBABILITY WITH AN EXPONENTIAL LÉVY PROCESS INVESTMENT RETURN AND HEAVY-TAILED CLAIMS
}

\author{
C. C. HEYDE, ${ }^{*}$ Australian National University and Columbia University \\ DINGCHENG WANG, ${ }^{* *}$ Australian National University and \\ University of Electronic Science and Technology of China
}

\begin{abstract}
By expressing the discounted net loss process as a randomly weighted sum, we investigate the finite-time ruin probabilities for the Poisson risk model with an exponential Lévy process investment return and heavy-tailed claims. It is found that in finite time, however, the extreme of insurance risk dominates the extreme of financial risk, but, for the case of dangerous investment (see Klüppelberg and Kostadinova (2008) for an accurate definition of dangerous investment), the extreme of financial risk has more and more of an effect on the total risk, and as time passes, the extreme of financial risk finally dominates the extreme of insurance risk.
\end{abstract}

Keywords: Finite-time ruin probability; investment return; Lévy process; Poisson risk model; self-financing portfolio; regularly varying tail; claim

2000 Mathematics Subject Classification: Primary 60G51; 62P05

Secondary 91B28; 91B30

\section{Introduction}

In the paper we investigate the finite-time ruin probability of a Poisson risk model with heavytailed claims and the risky investment of reserve. In the model, claims, $X_{n}, n \geq 1$, constitute a sequence of independent, identically distributed (i.i.d.), and positive random variables with a common distribution function (DF) $F$ such that $\bar{F}(x)=1-F(x)>0$ for all $x>0$. Let the arrival times $\tau_{1}, \tau_{2}, \ldots, \tau_{n}, \ldots$ constitute a homogeneous Poisson process

$$
N(t)=\sum_{n=1}^{\infty} \mathbf{1}_{\left[\tau_{n} \in[0, t]\right]}, \quad t \geq 0
$$

(here and in the sequel, $\mathbf{1}_{A}$ denotes the indicator function of the set $A$ ), which is independent of the sequence $X_{n}, n \geq 1$, where, by convention, $\tau_{0}=0$. Hence, the total amount of claims up to $t \geq 0$ can be written as

$$
S(t)=\sum_{n=1}^{N(t)} X_{n}
$$

Received 3 April 2008; revision received 19 December 2008.

* C. C. Heyde died in March 2008.

** Postal address: Center of Financial Mathematics, Mathematical Sciences Institute, Australian National University, Canberra, ACT 0200, Australia. Email address: dingcheng.wang@anu.edu.au 
with $S(t)=0$ when $N(t)=0$. If $N(t)$ follows a homogeneous Poisson process with intensity $\lambda>0$, then $\tau_{n}$ has a gamma distribution with probability density function

$$
f_{n}(t)=\frac{\lambda^{n} t^{n-1}}{(n-1) !} \mathrm{e}^{-\lambda t}, \quad t>0 .
$$

The total amount of premiums accumulated up to time $t \geq 0$ is denoted by $C(t)=c t$. Assume that an insurer invests its reserve into a Black-Scholes-type market consisting of a bond with a constant riskless interest rate $\delta>0$ and some stock, modelled by an exponential Lévy process, whose respective price processes follow the equations

$$
X_{0}(t)=\mathrm{e}^{\delta t} \quad \text { and } \quad X_{1}(t)=\mathrm{e}^{L(t)}, \quad t \geq 0 .
$$

The constant $\delta>0$ is the riskless interest rate. The process $\left\{(L(t))_{t \geq 0}\right\}$ is a Lévy process with characteristic exponent $\check{\Psi}$, i.e. $\mathrm{E}\left[\mathrm{e}^{\mathrm{i} s L(t)}\right]=\mathrm{e}^{t \check{\Psi}(s)}, s \in \mathbb{R}$ and $t \geq 0$, where $\breve{\Psi}$ has the Lévy-Khintchine representation

$$
\check{\Psi}(s)=\mathrm{i} s \gamma-\frac{\sigma^{2}}{2} s^{2}+\int_{\mathbb{R}}\left(\mathrm{e}^{\mathrm{i} s x}-1-\mathrm{i} s x \mathbf{1}_{[|x| \leq 1]}\right) \nu(\mathrm{d} x), \quad s \in \mathbb{R},
$$

where $\gamma \in \mathbb{R}, \sigma \geq 0$, and the Lévy measure $v$ satisfies $v(0)=0$ and $\int_{\mathbb{R}}\left(x^{2} \wedge 1\right) v(\mathrm{~d} x)<\infty$. The characteristic triplet $\left(\gamma, \sigma^{2}, v\right)$ determines the Lévy process. For more details, see Sato (1999).

Assume that an insurer uses the so-called constant mix strategy to invest their reserve, namely, at each instant of time an initially fixed fraction $\theta \in[0,1]$ of the wealth is invested in the risky asset and a fraction $1-\theta$ is invested in the riskless asset; see, e.g. Emmer et al. (2001) and Emmer and Klüppelberg (2004). This strategy is dynamic in the sense that it requires a rebalance of the portfolio at any moment of time depending on the corresponding price changes. This approach is based on self-financing portfolios and, hence, is classical in financial portfolio optimization; see Korn (1997, Section 2.1). The fraction $\theta$ is called the investment strategy.

Let $\{L(t) ; t \geq 0\}$ be a Lévy process with characteristic triplet $\left(\gamma, \sigma^{2}, v\right)$. Denote by $\epsilon(L)$ the solution of the following equation:

$$
\mathrm{d} Z(t)=Z(t-) \mathrm{d} L(t), \quad Z(0)=1 .
$$

Then Proposition 8.21 of Cont and Tankov (2004) gives the existence and uniqueness of $\varepsilon(L)$.

Next we follow the method used by Klüppelberg and Kostadinova (2008) to introduce the integrated risk process (IRP) as the result of the insurance business and the net gains of the investment through a stochastic differential equation (SDE).

Definition 1.1. For an investment strategy $\theta \in[0,1]$, we call the solution to the SDE

$$
\mathrm{d} U_{\theta}(t)=c \mathrm{~d} t-\mathrm{d} S(t)+U_{\theta}(t-) \mathrm{d} \hat{L}_{\theta}(t), \quad t \geq 0, U_{\theta}(0)=x,
$$

the IRP, where $\mathrm{d} \hat{L}_{\theta}(t)=(1-\theta) \delta \mathrm{d} t+\theta \mathrm{d} \hat{L}(t), \hat{L}(t)$ satisfies $\epsilon(\hat{L})=\mathrm{e}^{L(t)}, x>0$ is the initial capital, $c>0$ is the constant premium rate, $S(t)$ is the total claim amount process, and $\delta>0$ is the riskless interest rate.

Provided that the insurance and the investment processes are independent, by Lemma 2.2 of Klüppelberg and Kostadinova (2008), the solution to the SDE (1.1) is

$$
U_{\theta}(t)=\exp \left(L_{\theta}(t)\right)\left(x+\int_{0}^{t} \exp \left(-L_{\theta}(v)\right)(c \mathrm{~d} v-\mathrm{d} S(v))\right),
$$


where $L_{\theta}$ satisfies $\exp \left(L_{\theta}\right)=\epsilon\left(\hat{L}_{\theta}\right)$. By Lemma 2.5 of Emmer and Klüppelberg (2004), the $L_{\theta}(t)$ is a Lévy process with characteristic exponent $\psi_{\theta}$ and characteristic triplet $\left(\gamma_{\theta}, \sigma_{\theta}^{2}, v_{\theta}\right)$, which is specified by the original process $L$ in the following way:

$$
\begin{aligned}
\gamma_{\theta}= & \gamma \theta+(1-\theta)\left(\delta+\frac{\sigma^{2}}{2} \theta\right) \\
& +\int_{\mathbb{R}}\left(\log \left(1+\theta\left(\mathrm{e}^{x}-1\right)\right) \mathbf{1}_{\left[\left|\log \left(1+\theta\left(\mathrm{e}^{x}-1\right)\right)\right| \leq 1\right]}-\theta x \mathbf{1}_{[|x| \leq 1]}\right) \nu(\mathrm{d} x), \\
\sigma_{\theta}^{2}= & \theta^{2} \sigma^{2}, \\
\nu_{\theta}(A)= & v\left(\left\{x \in \mathbb{R}: \log \left(1+\theta\left(\mathrm{e}^{x}-1\right)\right) \in A\right\}\right) \quad \text { for any Borel set } A \subset \mathbb{R} .
\end{aligned}
$$

Thus, the IRP $U_{\theta}$ fits into the framework of generalized Ornstein-Uhlenbeck processes, which have recently attracted a lot of attention; see, e.g. Lindner and Maller (2005) or Carmona et al. (2001).

Denote the Laplace exponents of the processes $L$ and $L_{\theta}$ by

$$
\psi(s)=\log \mathrm{E}\left[\mathrm{e}^{-s L(1)}\right] \quad \text { and } \quad \psi_{\theta}(s)=\log \mathrm{E}\left[\exp \left(-s L_{\theta}(1)\right)\right],
$$

provided that they exist. If $\psi(s)<\infty$ then $\mathrm{E}\left[\mathrm{e}^{-s L(t)}\right]=\mathrm{e}^{t \psi(s)}<\infty$ for all $t \geq 0$; see Sato (1999, Theorem 25.17). From the proof of Lemma 4.1 of Klüppelberg and Kostadinova (2008) we can obtain $\psi_{\theta}(s)<\infty$ for all $\theta \in(0,1)$ and $s \in \mathbb{R}^{+}$, and if $0<\mathrm{E}[L(1)]<\infty$ and either $\sigma>0$ or $\nu((-\infty, 0))>0$, then, for $\theta \in(0,1)$, there exists a unique positive $\kappa=\kappa_{\theta}>0$ such that $\psi_{\theta}(\kappa)=0$.

Let us denote the discounted net loss process by

$$
V_{\theta}(t)=x-\exp \left(-L_{\theta}(t)\right) U_{\theta}(t)=\int_{0}^{t} \exp \left(-L_{\theta}(v)\right)(\mathrm{d} S(v)-c \mathrm{~d} \nu), \quad t \geq 0 .
$$

Now we can define the ruin probability up to time $T$ and the ultimate ruin probability, respectively, by

$$
\Psi(x, T)=\mathrm{P}\left\{V_{\theta}(t)>x \text { for some } 0 \leq t \leq T\right\}
$$

and

$$
\Psi(x)=\mathrm{P}\left\{V_{\theta}(t)>x \text { for some } \infty>t \geq 0\right\} .
$$

Many researchers have studied Poisson risk models with risky investment, usually modelled by a geometric Brownian motion. For example, Frolova et al. (2002) discussed the ultimate ruin probability with exponentially distributed claims and the risky asset of a constant fraction of the wealth; Paulsen and Gjessing (1997) and Kalashnikov and Norberg (2002) extended their result to light-tailed claim size distributions; Gaier and Grandits (2002) discussed the case of regularly varying claim size distribution.

An important question often asked is whether geometric Brownian motion can appropriately describe the change of prices of the risky assets. Many empirical investigations of stock markets have indicated that the prices of many stocks have sudden downward (or upward) jumps, which cannot be explained by the continuous geometric Brownian motion. A more suitable approach is to model the price of the risky asset by a general exponential Lévy process with jumps. Using this approach, Emmer and Klüppelberg (2004) have investigated the optimization problem of investment portfolios. Paulsen (2002) considered the asymptotic behaviour for large initial capital of the ultimate ruin probability for the case where the investment process is a general 
Lévy process. His result showed that the ruin probability behaves like a Pareto function of the (large) initial capital. The Pareto exponent depends on the interaction between the insurance claims and the investment process. Using the theory of stochastic recurrence equations (see Goldie (1991) and Konstantinides and Mikosch (2005)), Klüppelberg and Kostadinova (2008) studied the tail behaviour of the ultimate integrated risk for the discounted net loss process, namely, $\mathrm{P}\left\{V_{\theta}^{\infty}>x\right\}$, where $V_{\theta}^{\infty}=\lim _{t \rightarrow \infty} V_{\theta}(t)$. They showed that if the order of the finite moment of the claim size distribution is greater than $\kappa_{\theta}$ then the extreme of financial risk determines the tail behaviour of the ultimate integrated risk for the discounted net loss process. They referred to this as the case of dangerous investment. If the distribution function of claim sizes has a regularly varying tail with tail index $-\alpha<0$ and $\alpha<\kappa_{\theta}$, then they showed that the extreme of insurance risk determines the tail behaviour of the ultimate integrated risk for the discounted net loss process. They referred to this as the case of dangerous claims.

All the above papers pay attention to the ultimate ruin probability and the tail behaviour of the ultimate integrated risk process with the claim arrival process being a Poisson process. To the authors' knowledge, no other paper has addressed the finite-time ruin probability with risky investment. In fact, insurers pay more attention to their future finite-time risk, for example, five years, ten years, and can change initial capitals by introducing new stock holders or adding the shares of their companies and adding or decreasing premiums according to the insurers' business situations. So, finite-time ruin probability is a more realistic model. But it is often more difficult to investigate finite-time ruin probability. In this paper, by expressing the discounted net loss process as a randomly weighted sum we investigate the finite-time ruin probability for the integrated risk process under the Poisson risk model with heavy-tailed claims. We find that in finite time, however, the extreme of insurance risk dominates the extreme of financial risk, but, for the case of dangerous investment, extreme of financial risk has more and more of an effect on the total risk, and as time passes, the extreme of financial risk finally dominates the extreme of insurance risk.

The remaining part of this paper consists of two sections. In Section 2 we introduce some notation and provide the main result of the paper. Section 3 contains the proof of the main result.

\section{Notation and main result}

First of all, we recall several classes of heavy-tailed distributions, which are crucial for our purpose. We say that a distribution function $F$ has a regularly varying tail with tail index $-\alpha<0$, denoted by $F \in \mathcal{R}_{-\alpha}$, if there is some slowly varying function $L(\cdot)$ such that

$$
\bar{F}(x)=x^{-\alpha} L(x), \quad x>0 .
$$

We say that a DF $F$ belongs to the class $\mathscr{D}$ (has dominated variation) if, for any $0<l<1$ (or, equivalently, for some $0<l<1$ ),

$$
\limsup _{x \rightarrow \infty} \frac{\bar{F}(l x)}{\bar{F}(x)}<\infty .
$$

By definition we say that a DF $F$ belongs to the subexponential class of distributions, denoted by $s$, if $\bar{F}(x)=1-F(x)>0$ for all $x>0$ and the relation

$$
\lim _{x \rightarrow \infty} \frac{\bar{F}^{* n}(x)}{\bar{F}(x)}=n
$$


holds for any (or, equivalently, for some) $n \geq 2$, where $F^{* n}$ denotes the $n$-fold convolution of $F$.

We say that a DF $F$ belongs to the class $\mathcal{L}$ (is long tailed) if, for any $L>0$,

$$
\lim _{x \rightarrow \infty} \frac{\bar{F}(x+L)}{\bar{F}(x)}=1 .
$$

It is well known that

$$
\mathcal{R}_{-\alpha} \subset \mathscr{D} \cap \& \subset \& \subset \mathcal{L}
$$

see Embrechts et al. (1997) for details. We remark that $\mathscr{D} \cap \&$ is a useful subclass of the class $\&$. Specifically, it contains the famous class $\mathcal{R}$ of DFs with regular variation.

Now we recall two significant indices of a general random variable. Let $X$ be a random variable with a distribution function $F$ concentrated on $(-\infty, \infty)$. For any $y>0$, we set

$$
\bar{F}_{*}(y)=\liminf _{x \rightarrow \infty} \frac{\bar{F}(x y)}{\bar{F}(x)} \quad \text { and } \quad \bar{F}^{*}(y)=\limsup _{x \rightarrow \infty} \frac{\bar{F}(x y)}{\bar{F}(x)},
$$

and then we define

$$
\begin{aligned}
& \mathbb{J}_{F}^{+}=\mathbb{J}^{+}(X)=\inf \left\{-\frac{\log \bar{F}_{*}(y)}{\log y}: y>1\right\}=-\lim _{y \rightarrow \infty} \frac{\log \bar{F}_{*}(y)}{\log y}, \\
& \mathbb{J}_{F}^{-}=\mathbb{J}^{-}(X)=\sup \left\{-\frac{\log \bar{F}^{*}(y)}{\log y}: y>1\right\}=-\lim _{y \rightarrow \infty} \frac{\log \bar{F}^{*}(y)}{\log y} .
\end{aligned}
$$

Here $\mathbb{J}_{F}^{+}$and $\mathbb{J}_{F}^{-}$are called the upper and lower Matuszewska indices of the nonnegative and nondecreasing function $f(x)=(\bar{F}(x))^{-1}, x \geq 0$ (see Bingham et al. (1987, Chapter 2.1)). Specifically, if $F \in \mathscr{D}$ then $\mathbb{J}_{F}^{+}<\infty$; if $F \in \mathcal{R}_{-\alpha}$ with $\alpha \geq 0$ then $\mathbb{J}_{F}^{-}=\mathbb{J}_{F}^{+}=\alpha$.

It is well known that if $F \in \mathscr{D}$ then by Proposition 2.2.1 of Bingham et al. (1987) we know that, for $p_{2}>\mathbb{J}_{F}^{+}$, there are positive constants $C_{2}$ and $D_{2}$ such that

$$
\frac{\bar{F}(y)}{\bar{F}(x)} \leq C_{2}\left(\frac{x}{y}\right)^{p_{2}} \text { holds for all } x \geq y \geq D_{2} .
$$

In the sequel, $C$ always represents a positive constant, which is independent of $x$ and may vary from place to place. For two functions $a(\cdot)$ and $b(\cdot)$, with $b(\cdot)$ positive, satisfying

$$
\limsup _{x \rightarrow \infty} \frac{a(x)}{b(x)}<\infty
$$

we write $a(x)=O(b(x))$; if

$$
\lim _{x \rightarrow \infty} \frac{a(x)}{b(x)}=1
$$

we write $a(x) \sim b(x)$.

In this paper we only consider the case in which $\theta \in(0,1)$ because it is impossible for insurers to invest all the capital into risky assets and the pure bond strategy has been discussed in previous papers (see, e.g. Wang (2008)). Now we are ready to state the main result of this paper. 
Theorem 2.1. Consider the risk model introduced in Section 1 with $F \in \mathscr{D} \cap$ \&. Assume that $\theta \in(0,1)$. Then, we have, for every fixed $T>0$,

$$
\Psi(x, T) \sim \lambda \int_{0}^{T} \mathrm{P}\left\{X_{1} \exp \left(-L_{\theta}(t)\right)>x\right\} \mathrm{d} t, \quad x \rightarrow \infty .
$$

Specifically, if $F \in \mathcal{R}_{-\alpha}$ with $\alpha>0$ then we obtain, for every fixed $T>0$,

$$
\Psi(x, T) \sim \frac{\lambda}{-\psi_{\theta}(\alpha)}\left(1-\exp \left(\psi_{\theta}(\alpha) T\right)\right) \bar{F}(x), \quad x \rightarrow \infty .
$$

Remark. Let us consider the case in which claims have regularly varying tails. Theorem 4.4 of Klüppelberg and Kostadinova (2008) tells us that, for the case of dangerous investment, the extreme of financial risk finally dominates the extreme of insurance risk (for the case of dangerous investment, $\psi_{\theta}(\alpha)>0$ since $\alpha>\kappa_{\theta}$ and $\psi_{\theta}(\alpha)$ is a convex function with $\psi_{\theta}(0)=0$ and $\left.\psi_{\theta}^{\prime}(0)<0\right)$. In our Theorem 2.1, we do not suppose the existence of $\kappa_{\theta}$. Theorem 2.1 shows that in finite time, however, the extreme of insurance risk dominates the extreme of financial risk even for the case of dangerous investment. It is an interesting phenomenon. The phenomenon shows that in the case of dangerous investment the financial risk has more and more of an effect on the total risk as time passes, but in finite time the insurance risk still has a dominant effect on the total risk. This can be seen from (2.2). In the case of dangerous investment, financial risk finally dominates insurance risk as the cumulation of financial risk. It is shown from (2.2) that

$$
\frac{\lambda}{-\psi_{\theta}(\alpha)}\left(1-\exp \left(\psi_{\theta}(\alpha) T\right)\right) \rightarrow \infty \quad \text { as } T \rightarrow \infty,
$$

and in Theorem 4.4 of Klüppelberg and Kostadinova (2008), financial risk finally dominates insurance risk.

In the following we will give some examples.

Example 2.1. (Modelling the risky investment process by geometric Brownian motion with jumps and letting the distribution of the claim sizes have regularly varying tails.) Assume that the log returns of the risky asset are modelled as

$$
L(t)=v t+\sigma W(t)+K(t), \quad t \geq 0,
$$

where $v \in \mathbb{R}, \sigma>0,(W(t))_{t \geq 0}$ is a standard Brownian motion, and $K(t)=\sum_{n=1}^{M(t)} Y_{n}, t \geq 0$, is given by a homogeneous Poisson process $M(t)$ with intensity $\mu$ and i.i.d. jump sizes $\left\{Y_{n} ; n \geq 1\right\}$ with the generic random variable $Y$. Hence, the Laplace exponent of $L$ is given by

$$
\psi(s)=-v s+\sigma^{2} \frac{s^{2}}{2}+\mu\left(\mathrm{E}\left[\mathrm{e}^{-s Y}\right]-1\right) .
$$

Let the claim sizes constitute a sequence of i.i.d. random variables with regularly varying tail $\mathcal{R}_{-\alpha}, \alpha>0$. Hence, according to Theorem 2.1, we obtain

$$
\Psi(x, T) \sim \frac{\lambda}{-\psi_{\theta}(\alpha)}\left(1-\exp \left(\psi_{\theta}(\alpha) T\right)\right) \bar{F}(x), \quad x \rightarrow \infty,
$$

where, by (1.2), (1.3), and (1.4), we have

$$
\psi_{\theta}(\alpha)=-v_{\theta} \alpha+\sigma_{\theta}^{2} \frac{\alpha^{2}}{2}+\mu\left(\mathrm{E}\left[\left(1+\theta\left(\mathrm{e}^{Y}-1\right)\right)^{-\alpha}\right]-1\right),
$$

where $v_{\theta}=v \theta+(1-\theta)\left(\delta+\sigma^{2} \theta / 2\right)$ and $\sigma_{\theta}^{2}=\theta^{2} \sigma^{2}$. In particular, for the case in which the 
$\log$ returns of the risky asset are modelled by classical geometric Brownian motion, namely, $K(t) \equiv 0$, we have

$$
\psi_{\theta}(\alpha)=-v \theta \alpha-(1-\theta) \delta \alpha+\frac{\sigma^{2} \theta \alpha}{2}(-1+\theta+\theta \alpha) .
$$

Kou (2002) considered the case in which $Y$ has a double-exponential distribution. For this case, $Y$ has density function

$$
f(x)=f_{Y}(y)=p \eta_{1} \exp \left(-\eta_{1} y\right) \mathbf{1}_{[y \geq 0]}+(1-p) \eta_{2} \exp \left(\eta_{2} y\right) \mathbf{1}_{[y<0]}, \quad x \in \mathbb{R},
$$

where $\eta_{1}>0, \eta_{2}>0$, and $p \in(0,1)$. Hence,

$$
\psi_{\theta}(\alpha)=-v_{\theta} \alpha+\sigma_{\theta}^{2} \frac{\alpha^{2}}{2}+\mu \int_{\mathbb{R}}\left(\left(1+\theta\left(\mathrm{e}^{x}-1\right)\right)^{-\alpha}-1\right) f(x) \mathrm{d} x .
$$

Example 2.2. (Modelling the risky investment process by the variance-gamma Lévy process and letting the distribution of the claim sizes have regularly varying tails.) Let the claim size be distributed by $\mathcal{R}_{-\alpha}, \alpha>0$, and let the $\log$ return of the risky asset be modelled by the variance-gamma (VG) process, as suggested in Madan and Seneta (1990). A nonsymmetric VG model is given by

$$
L(t)=\mu t+W(K(t)), \quad t \geq 0,
$$

where $\mu>0, W$ is a Brownian motion with drift $a<0$ and variance $b^{2}, K(t)$ is a gamma Lévy process independent of $W$ and satisfies $K(1)$ has the same distribution as $\Gamma(\eta, r)$ whose density is given by

$$
f_{\Gamma}(x)=\frac{r^{\eta} x^{\eta-1} \mathrm{e}^{-r x}}{\Gamma(\eta)}, \quad x \geq 0,
$$

for parameters $r, \eta>0$. The characteristic triplet of $K$ is $\left(0,0, v_{\Gamma}\right)$ with

$$
\nu_{\Gamma}=\mathbf{1}_{[x>0]} \eta x^{-1} \mathrm{e}^{-r x} \mathrm{~d} x .
$$

The Laplace exponent of $L$ is

$$
\psi(s)=-\mu s-\eta \log \left(1-\frac{1}{r}\left(b^{2} \frac{s^{2}}{2}-s a\right)\right), \quad s \in \mathbb{R} .
$$

The Lévy measure of $L$ is given by

$$
v(\mathrm{~d} x)=\frac{r^{2}}{\eta|x|} \exp \left(\frac{a x}{b^{2}}-\frac{\sqrt{a^{2}+2 b^{2} r^{2} / \eta}|x|}{b^{2}}\right) \mathrm{d} x, \quad x \in \mathbb{R} .
$$

Hence, according to Theorem 2.1, we can obtain

$$
\Psi(x, T) \sim \frac{\lambda}{-\psi_{\theta}(\alpha)}\left(1-\exp \left(\psi_{\theta}(\alpha) T\right)\right) \bar{F}(x), \quad x \rightarrow \infty,
$$

where, by (1.2), (1.3), and (1.4), we have

$$
\begin{aligned}
\psi_{\theta}(\alpha)= & -\mu_{\theta} \alpha+\int_{-\infty}^{\infty}\left(\mathrm{e}^{-\alpha x}-1\right) v_{\theta}(\mathrm{d} x) \\
= & -\theta \mu \alpha-(1-\theta) \delta \alpha \\
& +\int_{x>\log (1-\theta)}\left(\left(1+\theta\left(\mathrm{e}^{x}-1\right)\right)^{-\alpha}-1\right) \frac{r^{2}}{\eta|x|} \exp \left(\frac{a x}{b^{2}}-\frac{\sqrt{a^{2}+2 b^{2} r^{2} / \eta}|x|}{b^{2}}\right) \mathrm{d} x .
\end{aligned}
$$

For more details, see Cont and Tankov (2004). 
As $\theta$ is fixed, $\psi_{\theta}(\alpha)$ is a convex function with $\psi_{\theta}(0)=0$ and $\mathrm{d} \psi_{\theta}(\alpha) / \mathrm{d} \alpha<0$, which means that at small $\alpha$, the index of the tail of insurance risk, insurance risk always dominates financial risk and, as $\alpha$ exceeds a threshold $\kappa_{\theta}$, financial risk will finally dominate insurance risk. As $\alpha$ is fixed, $\psi_{\theta}(\alpha)$ is an increasing function. From Table 1 and Table 2 we know that, when $\theta \geq 0.75$, for almost all $\alpha>0, \psi_{\theta}(\alpha)$ is positive, i.e. it is a dangerous investment; if $\theta=0.25$ then $\psi_{\theta}(\alpha)<0$ for $\alpha \leq 40$, which means that insurance risk dominates financial risk. Hence, it is dangerous for insurers to invest a large fraction of their wealth in the risky asset.

TABLE 1: $\psi_{\theta}(\alpha)$ for the model in Kou (2002) with $p=0.3, \eta_{1}=50$, and $\eta_{2}=25$ for the parameters of $Y$, and using the following generic stock return figures: a mean of 15 per year, a standard deviation of 20 per year, a jump rate of 10 per year (giving a jump rate of 10/250 per day when converted into daily rates for the calculations), and a riskless interest rate of $10 \%$ per year.

\begin{tabular}{rcrc}
\hline$\alpha$ & $\theta=0.25$ & $\theta=0.5$ & \multicolumn{1}{c}{$\theta=0.75$} \\
\hline 1 & -0.00022239054 & -0.00005142036 & 0.00013320982 \\
2 & -0.00043886108 & -0.00007716072 & 0.00032545964 \\
3 & -0.00064925162 & -0.00007618108 & 0.00058158946 \\
4 & -0.00085356216 & -0.00002552144 & 0.00090715928 \\
5 & -0.00105127270 & 0.00000689820 & 0.00130884910 \\
6 & -0.00124262324 & 0.00009387784 & 0.00179281892 \\
7 & -0.00142725378 & 0.00021381748 & 0.00236842874 \\
8 & -0.00160360432 & 0.00036711712 & 0.00304775856 \\
9 & -0.00177429486 & 0.00055761676 & 0.00383304838 \\
10 & -0.00193754539 & 0.00078619640 & 0.00474621820 \\
20 & -0.00312803027 & 0.00589795505 & 0.02592153780 \\
30 & -0.00332426826 & 0.02020884429 & 0.1370653366 \\
40 & -0.00214341530 & 0.05939558138 & 2.478260297 \\
50 & 0.00102750319 & 0.1930346037 & 662.6718907 \\
\hline
\end{tabular}

TABLE 2: $\psi_{\theta}(\alpha)$ for the VG model using the following generic stock return figures: $\mu=0.0006246405$, standard deviation $b=0.01136155$, drift $a=-0.0003754359$, riskless interest rate $\delta=10 \%$ per year, and $r=1.005205$ for the parameters of $L$.

\begin{tabular}{rlcl}
\hline$\alpha$ & \multicolumn{1}{c}{$\theta=0.25$} & $\theta=0.5$ & $\theta=0.75$ \\
\hline 1 & -0.000417916 & -0.000315274 & -0.000197204 \\
2 & -0.000826290 & -0.000604616 & -0.000331239 \\
3 & -0.001227325 & -0.000858451 & -0.000380516 \\
4 & -0.001626658 & -0.001073567 & -0.000361672 \\
5 & -0.002013134 & -0.001261096 & -0.000273805 \\
6 & -0.002391529 & -0.001416231 & -0.00109617 \\
7 & -0.002756264 & -0.001542656 & 0.000127846 \\
8 & -0.003117696 & -0.001633436 & 0.000438739 \\
9 & -0.003471038 & -0.001691719 & 0.000823237 \\
10 & -0.003816291 & -0.001717466 & 0.001281529 \\
20 & -0.006828308 & -0.000177407 & 0.00997225 \\
30 & -0.009023775 & 0.0046861 & 0.02638223 \\
40 & -0.01040273 & 0.0129668 & 0.05100433 \\
50 & -0.0109496 & 0.02479714 & 0.08456577 \\
\hline
\end{tabular}


Examples 2.1 and 2.2 were also considered in Klüppelberg and Kostadinova (2008). Next we give another example.

Example 2.3. (Modelling the risky investment process by a skewed $\beta / 2$-stable process and letting the distribution of the claim sizes have regularly varying tails.) In Example 2.2, instead of a gamma Lévy process, we supposed that $K(t)$ was a scaled, maximally skewed $\beta / 2$-stable Lévy process. This asset price model was considered in Hurst et al. (1999). A scaled, maximally skewed $\beta / 2$-stable Lévy process is a process with stationary nonnegative independent increments,

$$
K(t+s)-K(t) \sim S_{\beta / 2}\left(c s^{\beta / 2}, 1,0\right), \quad s, t \geq 0,
$$

where $\beta \in(0,2)$. Hence, the increment $K(t+s)-K(t)$ has an index of stability less than 1 , maximal skewness of 1 , zero location parameter, and characteristic function

$$
\exp \left(-s c^{\beta / 2}|y|^{\beta / 2}\left(1-\mathrm{i} \tan \left(\frac{\pi \beta}{4}\right) \operatorname{sgn}(y)\right)\right), \quad y \in \mathbb{R} .
$$

According to Theorem 2.1, we obtain

$$
\Psi(x, T) \sim \frac{\lambda}{-\psi_{\theta}(\alpha)}\left(1-\exp \left(\psi_{\theta}(\alpha) T\right)\right) \bar{F}(x), \quad x \rightarrow \infty .
$$

Next we calculate $\psi_{\theta}(\alpha)$. Because the increment $K(t+s)-K(t)$ is a stable process with stationary, nonnegative independent increments, we find that the generating triplet of the Lévy process is $(0,0, \rho)$ with

$$
\rho(\mathrm{d} r)=\frac{c_{1} \mathrm{~d} r}{r^{1+\beta / 2}}, \quad r>0,
$$

where $\lambda$ is some positive constant. Therefore, according to Theorem 30.1 of Sato (1999), we find that $W(K(t))$ is a Lévy process with generating triplet $(0,0, v)$ such that

$$
\begin{aligned}
v(\mathrm{~d} x) & =\int_{0}^{\infty} \frac{1}{\sqrt{2 \pi s}} \exp \left(-\frac{x^{2}}{2 s}\right) \frac{c_{1} \mathrm{~d} s}{s^{1+\beta / 2}} \mathrm{~d} x \\
& =\frac{c_{1} 2^{\beta / 2}}{\sqrt{\pi}} \Gamma\left(\frac{\beta+1}{2}\right) \frac{\mathrm{d} x}{|x|^{1+\beta}}, \quad-\infty<x<\infty .
\end{aligned}
$$

Now we calculate $c_{1}$. Clearly, by the uniqueness of the characteristic function and Lemma 14.11 of Sato (1999), we have

$$
\begin{aligned}
& -c^{\beta / 2}|y|^{\beta / 2}\left(1-\mathrm{i} \tan \left(\frac{\pi \beta}{4}\right) \operatorname{sgn}(y)\right) \\
& =\int_{\mathbb{R}}\left(\mathrm{e}^{\mathrm{i} y r}-1\right) \rho(\mathrm{d} r) \\
& =c_{1} \int_{0}^{\infty}\left(\mathrm{e}^{\mathrm{i} y r}-1\right) \frac{\mathrm{d} r}{r^{1+\beta / 2}} \\
& =c_{1} \Gamma\left(-\frac{\beta}{2}\right)|y|^{\beta / 2} \exp \left(-\mathrm{i} \frac{\pi \beta}{4} \operatorname{sgn}(y)\right) \\
& =c_{1} \Gamma\left(-\frac{\beta}{2}\right)|y|^{\beta / 2} \cos \left(\frac{\pi \beta}{4}\right)\left(1-\mathrm{i} \tan \left(\frac{\pi \beta}{4}\right) \operatorname{sgn}(y)\right) .
\end{aligned}
$$


Therefore,

$$
c_{1}=-\frac{c^{\beta / 2}}{\Gamma(-\beta / 2) \cos (\pi \beta / 4)} .
$$

By (1.2), (1.3), and (1.4), we have

$$
\begin{aligned}
\psi_{\theta}(\alpha)= & -\mu_{\theta} \alpha+\int_{-\infty}^{\infty}\left(\mathrm{e}^{-\alpha x}-1\right) v_{\theta}(\mathrm{d} x) \\
= & -\theta \mu \alpha-(1-\theta) \delta \alpha \\
& +\int_{x>\log (1-\theta)}\left(\left(1+\theta\left(\mathrm{e}^{x}-1\right)\right)^{-\alpha}-1\right) \frac{c_{1} 2^{\beta / 2}}{\sqrt{\pi}} \Gamma\left(\frac{\beta+1}{2}\right) \frac{\mathrm{d} x}{|x|^{1+\beta}} .
\end{aligned}
$$

\section{Proof of Theorem 2.1}

To prove Theorem 2.1, we start by establishing four necessary lemmas.

Let $X$ and $Y$ be two independent random variables, where $X$ is concentrated on $(-\infty, \infty)$ with a DF $F$ and $Y$ is a nonnegative random variable with $\mathrm{DF} G$, which is not degenerate at 0 . We write

$$
Z=X Y \text {, }
$$

and denote the distribution of $Z$ by $F \otimes G$. The lemma below is a direct consequence of Theorem 3.3(iv) of Cline and Samorodnitsky (1994) (see also Lemma 3.8 of Tang and Tsitsiashvili (2003a)).

Lemma 3.1. Consider the model in (3.1). If $F \in \mathscr{D} \cap \&$ and $\int_{0}^{\infty} x^{r} \mathrm{~d} G(x)<\infty$, where $\infty>r>\mathbb{J}_{F}^{+}$, then the distribution of the product $X Y$ belongs to the intersection $\mathscr{D} \cap \&$ and we have

$$
\bar{F} \otimes G(x) \asymp \bar{F}(x) .
$$

The proof of Lemma 3.2, below, parallels the argument in Tang and Tsitsiashvili (2004) with some adjustments.

Lemma 3.2. Suppose that a nonnegative random variable $X$ with $D F F \in \mathscr{D}$ and a nonnegative random variable $\xi$ are independent. Then, for arbitrarily fixed $\delta>0$ and $p>0$ such that $\mathbb{J}_{F}^{+}<p<\infty$, there exists some positive constant $C$ without relation to $\xi$ and $\delta$ such that, for all positive integers $i$ and all large $x$,

$$
\mathrm{P}\{X \xi>\delta x \mid \xi\} \leq C \bar{F}(x)\left(\delta^{-p} \xi^{p} \mathbf{1}_{[\delta \leq \xi]}+\mathbf{1}_{[\delta>\xi]}\right) .
$$

Proof. To avoid triviality, we assume that $\xi \neq 0$. We divide $\mathrm{P}\left\{X^{+} \xi>\delta x \mid \xi\right\}$ into the sum of the following three parts:

$$
\begin{gathered}
V_{1}(x)=\mathrm{P}\left\{X^{+} \xi>\delta x, \xi \geq \frac{\delta x}{D_{2}} \mid \xi\right\}, \\
V_{2}(x)=\mathrm{P}\left\{X^{+} \xi>\delta x, \frac{\delta x}{D_{2}}>\xi \geq \delta \mid \xi\right\}, \\
V_{3}(x)=\mathrm{P}\left\{X^{+} \xi>\delta x, \xi<\delta \mid \xi\right\},
\end{gathered}
$$

where $D_{2}$ is given in (2.1). Applying inequality (2.1), we find that

$$
V_{1}(x) \leq \mathbf{1}_{\left[\xi \geq \delta x / D_{2}\right]} \leq D_{2}^{p} \delta^{-p} x^{-p} \xi^{p} \mathbf{1}_{[\xi \geq \delta]} \leq C \delta^{-p} \bar{F}(x) \xi^{p} \mathbf{1}_{[\xi \geq \delta]}
$$


holds uniformly for large $x$. Applying inequality (2.1) again, we find that

$$
V_{2}(x) \leq C \delta^{-p} \bar{F}(x) \xi^{p} \mathbf{1}_{\left[\delta x / D_{2}>\xi \geq \delta\right]} \leq C \delta^{-p} \bar{F}(x) \xi^{p} \mathbf{1}_{[\xi \geq \delta]}
$$

holds uniformly for large $x$. We can also find that

$$
V_{3}(x) \leq \mathrm{P}\{X>x, \xi<\delta \mid \xi\}=\bar{F}(x) \mathbf{1}_{[\xi<\delta]}
$$

holds uniformly for all $x>0$. Hence, we conclude (3.2). This completes the proof.

Lemma 3.3. For the risk model introduced in Subsection 1.1, we have

$$
\sum_{j=1}^{\infty} \mathrm{P}\left\{X_{j} \exp \left(-L_{\theta}\left(\tau_{j}\right)\right) \mathbf{1}_{\left[\tau_{j} \leq T\right]}>y\right\}=\lambda \int_{0}^{T} \mathrm{P}\left\{X_{1} \exp \left(-L_{\theta}(t)\right)>y\right\} \mathrm{d} t .
$$

Proof. Let $G_{j}(t)$ denote the distribution function of $\tau_{j}$. Hence, we obtain

$$
\begin{aligned}
\sum_{j=1}^{\infty} \mathrm{P}\left\{X_{j} \exp \left(-L_{\theta}\left(\tau_{j}\right)\right) \mathbf{1}_{\left[\tau_{j} \leq T\right]}>y\right\} & =\sum_{j=1}^{\infty} \int_{0}^{T} \mathrm{P}\left\{X_{1} \exp \left(-L_{\theta}(t)\right)>y\right\} \mathrm{d} G_{j}(t) \\
& =\sum_{j=1}^{\infty} \int_{0}^{T} \mathrm{P}\left\{X_{1} \exp \left(-L_{\theta}(t)\right)>y\right\} \lambda \frac{(\lambda t)^{j-1}}{(j-1) !} \mathrm{e}^{-\lambda t} \mathrm{~d} t \\
& =\lambda \int_{0}^{T} \mathrm{P}\left\{X_{1} \exp \left(-L_{\theta}(t)\right)>y\right\} \mathrm{d} t .
\end{aligned}
$$

Lemma 3.4. Let $\left\{X_{k} ; 1 \leq k \leq n\right\}$ be $n$ i.i.d. random variables with common distribution function $F \in$ \&. Then, for any fixed $0<a \leq b<\infty$,

$$
\mathrm{P}\left\{\sum_{k=1}^{n} c_{k} X_{k}>x\right\} \sim \sum_{k=1}^{n} \mathrm{P}\left\{c_{k} X_{k}>x\right\}
$$

holds uniformly for all real numbers $\left\{c_{k} ; 1 \leq k \leq n\right\}$ such that $c_{k} \in[a, b], 1 \leq k \leq n$.

Proof. See Proposition 5.1 of Tang and Tsitsiashvili (2003b).

We are now in a position to give a proof of Theorem 2.1 .

Proof of Theorem 2.1. Clearly,

$$
V_{\theta}(t)=\sum_{n=1}^{\infty} X_{n} \exp \left(-L_{\theta}\left(\tau_{n}\right)\right) \mathbf{1}_{\left[\tau_{n} \leq t\right]}-c \int_{[0, t]} \exp \left(-L_{\theta}(y)\right) \mathrm{d} y, \quad t \geq 0 .
$$

Thus,

$$
\Psi(x, T) \leq \mathrm{P}\left\{\sum_{n=1}^{\infty} X_{n} \exp \left(-L_{\theta}\left(\tau_{n}\right)\right) \mathbf{1}_{\left[\tau_{n} \leq T\right]}>x\right\} .
$$

Klüppelberg and Kostadinova (2008) have shown that $\psi_{\theta}(s)<\infty$ for all $s>0$ and $0<\theta<1$ (see the proof of Lemma 4.1(a) in Klüppelberg and Kostadinova (2008)). Denote $\vartheta_{n}(t)=$ $\exp \left(-L_{\theta}\left(\tau_{n}\right)\right) \mathbf{1}_{\left[\tau_{n} \leq t\right]}$, and let $G_{j}$ be the DF of $\tau_{j}$. For every $\beta>0$, we have

$$
\sum_{n=1}^{\infty} \mathrm{E}\left[\vartheta_{n}^{\beta}(T)\right]=\sum_{n=1}^{\infty} \mathrm{E}\left[\int_{0}^{T} \exp \left(-\beta L_{\theta}(t)\right) \frac{\lambda(\lambda t)^{n-1}}{(n-1) !} \mathrm{e}^{-\lambda t} \mathrm{~d} t\right]=\lambda \int_{0}^{T} \exp \left(t \psi_{\theta}(\beta)\right) \mathrm{d} t<\infty .
$$


Take $p_{2}>\mathbb{J}_{F}^{+}$. We have, for every $\delta^{\prime}>0$,

$$
\begin{aligned}
& \sum_{n=1}^{\infty} n^{8 p_{2}+6}\left(\mathrm{E}\left[\vartheta_{n}^{2 p_{2}}(T)\right]+\mathrm{E}\left[\mathbf{1}_{\left[\vartheta_{n}(T)<\delta^{\prime}\right]}\right]\right) \\
& =\sum_{n=1}^{\infty} n^{8 p_{2}+6}\left(\mathrm{E}\left[\int_{0}^{T} \exp \left(-2 p_{2} L_{\theta}(t)\right) \mathrm{d} G_{n}(t)\right]\right. \\
& \left.\quad \quad+\int_{0}^{T} \mathrm{P}\left\{\exp \left(-L_{\vartheta}(t)\right)<\delta^{\prime}\right\} \mathrm{d} G_{n}(t)\right) \\
& =\sum_{n=1}^{\infty} n^{8 p_{2}+6}\left(\int_{0}^{T} \exp \left(t \psi_{\theta}\left(2 p_{2}\right)\right) \mathrm{d} G_{n}(t)+\int_{0}^{T} \mathrm{P}\left\{\exp \left(-L_{\vartheta}(t)\right)<\delta^{\prime}\right\} \mathrm{d} G_{n}(t)\right) \\
& \leq \max \left\{2, \exp \left(T \psi_{\theta}\left(2 p_{2}\right)\right)+1\right\} \sum_{n=1}^{\infty} n^{8 p_{2}+6} G_{n}(T) \\
& =\max \left\{2, \exp \left(T \psi_{\theta}\left(2 p_{2}\right)\right)+1\right\} \sum_{n=1}^{\infty} n^{8 p_{2}+6} \mathrm{P}\{N(T) \geq n\} \\
& \leq C \max \left\{2, \exp \left(T \psi_{\theta}\left(2 p_{2}\right)\right)+1\right\} \sum_{k=1}^{\infty} k^{8 p_{2}+7} \mathrm{P}\{N(T)=k\} \\
& <\infty
\end{aligned}
$$

Clearly, for every $m_{0}>1$, using the Cauchy-Schwartz inequality, we obtain

$$
\begin{aligned}
& \mathrm{E}\left[\left(\sum_{n=m_{0}+1}^{\infty} n^{4 p_{2}+2} \max \left\{\vartheta_{n}^{p_{2}}(T), \mathbf{1}_{\left[\vartheta_{n}(T)<1 / n^{2}\right]}\right\}\right)^{2}\right] \\
& \leq \mathrm{E}\left[\sum_{n=m_{0}+1}^{\infty} n^{-2}\right]\left(\sum_{n=m_{0}+1}^{\infty} n^{8 p_{2}+6} \max \left\{\vartheta_{n}^{2 p_{2}}(T), \mathbf{1}_{\left[\vartheta_{n}(T)<1 / n^{2}\right]}\right\}\right) \\
& \leq C \sum_{n=m_{0}+1}^{\infty} n^{8 p_{2}+6}\left(\mathrm{E}\left[\vartheta_{n}^{2 p_{2}}(T)\right]+\mathrm{E}\left[\mathbf{1}_{\left[\vartheta_{n}(T)<1 / n^{2}\right]}\right]\right) .
\end{aligned}
$$

Hence, for any $\varepsilon>0$, we can take $m_{0}=m_{0}(\varepsilon)$ large enough such that

$$
\begin{gathered}
m_{0}^{2 p_{2}+2} \mathrm{E}^{1 / 2}\left[\left(\sum_{n=m_{0}+1}^{\infty} \mathbf{1}_{\left[\vartheta_{n}(T)<1 / n^{2}\right]}\right)^{2}\right] \leq \mathrm{E}^{1 / 2}\left[\left(\sum_{n=m_{0}+1}^{\infty} n^{2 p_{2}+2} \mathbf{1}_{\left[\vartheta_{n}(T)<1 / n^{2}\right]}\right)^{2}\right]<\varepsilon \\
m_{0}^{2 p_{2}+2} \mathrm{E}^{1 / 2}\left[\left(\sum_{n=m_{0}+1}^{\infty} \vartheta_{n}^{p_{2}}(T) n^{2 p_{2}}\right)^{2}\right] \leq \mathrm{E}^{1 / 2}\left[\left(\sum_{n=m_{0}+1}^{\infty} \vartheta_{n}^{p_{2}}(T) n^{4 p_{2}+2}\right)^{2}\right]<\varepsilon \\
\sum_{n=m_{0}}^{\infty} \frac{1}{n^{2}}<1 .
\end{gathered}
$$

Denote the conditional distribution functions of $\sum_{n=1}^{m_{0}} \vartheta_{n}(T) X_{n}$ and $\sum_{n=m_{0}+1}^{\infty} \vartheta_{n}(T) X_{n}$, given 
$\left\{\vartheta_{n}(T), n \geq 1\right\}$, by $F_{1 \vartheta}$ and $F_{2 \vartheta}$, respectively. By Lemma 3.2 we obtain

$$
\begin{aligned}
\bar{F}_{2 \vartheta}(x) & \leq \mathrm{P}\left\{\sum_{n=m_{0}+1}^{\infty} \vartheta_{n}(T) X_{n}>\sum_{n=m_{0}+1}^{\infty} \frac{1}{n^{2}} x \mid \vartheta_{n}(T), n \geq 1\right\} \\
& \leq \sum_{n=m_{0}+1}^{\infty} \mathrm{P}\left\{\vartheta_{n}(T) X_{n}>\frac{1}{n^{2}} x \mid \vartheta_{n}(T), n \geq 1\right\} \\
& \leq C \bar{F}(x) \sum_{n=m_{0}+1}^{\infty}\left(\vartheta_{n}^{p_{2}}(T) n^{2 p_{2}}+\mathbf{1}_{\left[\vartheta_{n}(T)<1 / n^{2}\right]}\right) \\
& =C \bar{F}(x)\left(L_{\vartheta 1}\left(T, m_{0}\right)+L_{\vartheta 2}\left(T, m_{0}\right)\right) .
\end{aligned}
$$

Therefore, for some $M$ and $x \geq M$,

$$
\begin{aligned}
\Psi(x, T) \leq & \mathrm{E}\left[\mathrm{P}\left\{\sum_{n=1}^{m_{0}} \vartheta_{n}(T) X_{n}+\sum_{n=m_{0}+1}^{\infty} \vartheta_{n}(T) X_{n}>x \mid \vartheta_{n}(T), n \geq 1\right\}\right] \\
= & \mathrm{E}\left[\left(\int_{-\infty}^{x-M}+\int_{x-M}^{\infty}\right) \bar{F}_{2 \vartheta}(x-y) F_{1 \vartheta}(\mathrm{d} y)\right] \\
\leq & C \mathrm{E}\left[\left(L_{\theta 1}\left(T, m_{0}\right)+L_{\theta 2}\left(T, m_{0}\right)\right) \int_{-\infty}^{x-M} \bar{F}(x-y) F_{1 \vartheta}(\mathrm{d} y)\right] \\
& +\mathrm{E}\left[\int_{x-M}^{\infty} F_{1 \vartheta}(\mathrm{d} y)\right] \\
\leq & C \mathrm{E}\left[\left(L_{\theta 1}\left(T, m_{0}\right)+L_{\theta 2}\left(T, m_{0}\right)\right)\right. \\
& \left.\quad \times \mathrm{P}\left\{X_{m_{0}+1}+\sum_{n=1}^{m_{0}} \vartheta_{n}(T) X_{n}>x \mid \vartheta_{n}(T), n \geq 1\right\}\right] \\
& +\mathrm{P}\left\{\sum_{n=1}^{m_{0}} \vartheta_{n}(T) X_{n}>x-M\right\} \\
= & I_{1}(T)+I_{2}(T) .
\end{aligned}
$$

By the dominated convergence theorem we have

$$
\lim _{r \rightarrow 0} \mathrm{P}\left\{\vartheta_{i}(T)<r\right\}=\int_{0}^{T} \lim _{r \rightarrow 0} \mathrm{P}\left\{\exp \left(-L_{\theta}(t)\right)<r\right\} \mathrm{d} G_{i}(t)=0 .
$$

Therefore, by (3.3) we can take $1>\eta>0$ and $B>0$ such that, for all $j, n=1,2, \ldots, m_{0}$,

$$
\mathrm{E}\left[\vartheta_{n}^{p_{2}}(T) \mathbf{1}_{\left[\vartheta_{j}(T)<\eta\right]}\right]<\frac{\varepsilon}{m_{0}^{p_{2}+2}}, \quad \mathrm{P}\left\{\vartheta_{j}(T)<\eta\right\}<\frac{\varepsilon}{m_{0}^{p_{2}+2}},
$$

and

$$
\mathrm{E}\left[\vartheta_{n}^{p_{2}}(T) \mathbf{1}_{\left[\vartheta_{j}(T)>B\right]}\right]<\frac{\varepsilon}{m_{0}^{p_{2}+2}}, \quad \mathrm{P}\left\{\vartheta_{j}(T)>B\right\}<\frac{\varepsilon}{m_{0}^{p_{2}+2}}
$$


For $I_{2}(T)$, we obtain

$$
\begin{aligned}
I_{2}(T) \leq & \mathrm{P}\left\{\sum_{n=1}^{m_{0}} X_{n} \vartheta_{n}(T)>x-M, \bigcap_{1 \leq j \leq m_{0}}\left\{\vartheta_{j}(T) \leq B\right\} ; \bigcap_{1 \leq j \leq m_{0}}\left\{\vartheta_{j}(T) \geq \eta\right\}\right\} \\
& +\mathrm{P}\left\{\sum_{n=1}^{m_{0}} X_{n} \vartheta_{n}(T)>x-M, \bigcup_{1 \leq j \leq m_{0}}\left\{\vartheta_{j}(T)<\eta\right\} ; \bigcap_{1 \leq j \leq m_{0}}\left\{\vartheta_{j}(T) \leq B\right\}\right\} \\
& +\mathrm{P}\left\{\sum_{n=1}^{m_{0}} X_{n} \vartheta_{n}(T)>x-M, \bigcup_{1 \leq j \leq m_{0}}\left\{\vartheta_{j}(T)>B\right\}\right\} \\
= & I_{3}(T)+I_{4}(T)+I_{5}(T) .
\end{aligned}
$$

Now we treat $I_{4}(T)$. By Lemma 3.2, (2.1), the definition of $\eta$, and the property of the long tail, we have, for all large $x>M$,

$$
\begin{aligned}
I_{4}(T) & \leq \sum_{j=1}^{m_{0}} \mathrm{P}\left\{\sum_{n=1}^{m_{0}} X_{n} \vartheta_{n}(T)>x-M, \vartheta_{j}(T)<\eta\right\} \\
& \leq \sum_{j=1}^{m_{0}} \sum_{n=1}^{m_{0}} \mathrm{P}\left\{X_{n} \vartheta_{n}(T)>\frac{x-M}{m_{0}}, \vartheta_{j}(T)<\eta\right\} \\
& =\sum_{j=1}^{m_{0}} \sum_{n=1}^{m_{0}} \mathrm{E}\left[\mathbf{1}_{\left[\vartheta_{j}(T)<\eta\right]} \mathrm{P}\left\{X_{n} \vartheta_{n}(T)>\frac{x-M}{m_{0}} \mid \vartheta_{k}(T), 1 \leq k \leq m_{0}\right\}\right] \\
& \leq C \bar{F}(x-M) \sum_{j=1}^{m_{0}} \sum_{n=1}^{m_{0}} \mathrm{E}\left[\mathbf{1}_{\left[\vartheta_{j}(T)<\eta\right]}\left(m_{0}^{p_{2}} \vartheta_{n}^{p_{2}}(T)+\mathbf{1}_{\left[\vartheta_{n}(T)<1 / m_{0}\right]}\right)\right] \\
& \leq C m_{0}^{p_{2}} \bar{F}(x-M) \sum_{j=1}^{m_{0}} \sum_{n=1}^{m_{0}}\left(\mathrm{E}\left[\vartheta_{n}^{p_{2}}(T) \mathbf{1}_{\left[\vartheta_{j}(T)<\eta\right]}\right]+\mathrm{P}\left\{\vartheta_{j}(T)<\eta\right\}\right) \\
& \leq C \varepsilon \bar{F}(x) .
\end{aligned}
$$

Let $A_{1}=\bigcap_{1 \leq j \leq m_{0}}\left\{\eta \leq \vartheta_{j}(T) \leq B\right\}$. Using Lemma 3.4 and $F \in \mathscr{D} \cap \&$, and noting that $X_{n} \vartheta_{n}(T) \in \mathscr{D} \cap \&$ by Lemma 3.1 and (3.3), we derive

$$
\begin{aligned}
I_{3}(T)= & \mathrm{E}\left[\mathrm{P}\left\{\sum_{n=1}^{m_{0}} X_{n} \vartheta_{n}(T)>x-M, A_{1} \mid \vartheta_{n}(T), n=1, \ldots, m_{0}\right\}\right] \\
& \sim \sum_{n=1}^{m_{0}} \mathrm{E}\left[\mathrm{P}\left\{X_{n} \vartheta_{n}(T)>x-M, A_{1} \mid \vartheta_{n}(T), n=1, \ldots, m_{0}\right\}\right] \\
\leq & \sum_{n=1}^{m_{0}} \mathrm{P}\left\{X_{n} \vartheta_{n}(T)>x-M\right\} \\
& \sim \sum_{n=1}^{m_{0}} \mathrm{P}\left\{X_{n} \vartheta_{n}(T)>x\right\} .
\end{aligned}
$$


Next we treat $I_{5}(T)$. Similarly to $I_{4}(T)$ we have, for large $x$,

$$
\begin{aligned}
I_{5}(T) & \leq \sum_{j=1}^{m_{0}} \mathrm{P}\left\{\sum_{n=1}^{m_{0}} X_{n} \vartheta_{n}(T)>x-M, \vartheta_{j}(T)>B\right\} \\
& \leq C m_{0}^{p_{2}} \bar{F}(x-M) \sum_{j=1}^{m_{0}} \sum_{n=1}^{m_{0}}\left(\mathrm{E}\left[\vartheta_{n}^{p_{2}}(T) \mathbf{1}_{\left[\vartheta_{j}(T)>B\right]}\right]+\mathrm{P}\left\{\vartheta_{j}(T)>B\right\}\right) \\
& \leq C \varepsilon \bar{F}(x) .
\end{aligned}
$$

Combining (3.10)-(3.13) we obtain, for all large $x>0$,

$$
I_{2}(T) \leq C \varepsilon \bar{F}(x)+(1+\varepsilon) \sum_{n=1}^{\infty} \mathrm{P}\left\{X_{n} \vartheta_{n}(T)>x\right\} .
$$

Next we deal with $I_{1}(T)$. Clearly, by Lemma 3.2 and (2.1), we obtain, for large $x$,

$$
\begin{aligned}
\mathrm{P}\left\{X_{m_{0}+1}+\sum_{n=1}^{m_{0}} \vartheta_{n}(T) X_{n}>x \mid \vartheta_{n}(T), n \geq 1\right\} \\
\leq \sum_{n=1}^{m_{0}} \mathrm{P}\left\{\vartheta_{n}(T) X_{n}>\frac{x}{m_{0}+1} \mid \vartheta_{n}(T), n \geq 1\right\} \\
\quad+\mathrm{P}\left\{X_{n+1}>\frac{x}{m_{0}+1} \mid \vartheta_{n}(T), n \geq 1\right\} \\
\leq C \bar{F}\left(\frac{x}{m_{0}+1}\right) \sum_{n=1}^{m_{0}}\left(\vartheta_{n}^{p_{2}}(T)+\mathbf{1}_{\left[\vartheta_{n}(T)<1\right]}\right)+\bar{F}\left(\frac{x}{m_{0}+1}\right) \\
\leq C m_{0}^{p_{2}} \bar{F}(x)\left(m_{0}+1+\sum_{n=1}^{m_{0}} \vartheta_{n}^{p_{2}}(T)\right) \\
=L_{\vartheta 3}\left(T, m_{0}\right) \bar{F}(x) .
\end{aligned}
$$

Substituting (3.15) into $I_{1}(T)$ and using (3.5) and (3.6), we obtain, from the Cauchy-Schwartz inequality, for large $x$,

$$
\begin{aligned}
I_{1}(T) & \leq C \bar{F}(x) \mathrm{E}\left[\left(L_{\vartheta 1}\left(T, m_{0}\right)+L_{\vartheta 2}\left(T, m_{0}\right)\right) L_{\vartheta 3}\left(T, m_{0}\right)\right] \\
& \leq C \bar{F}(x) \mathrm{E}^{1 / 2}\left[L_{\vartheta 1}\left(T, m_{0}\right)+L_{\vartheta 2}\left(T, m_{0}\right)\right]^{2} \mathrm{E}^{1 / 2}\left[L_{\vartheta 3}^{2}\left(T, m_{0}\right)\right] \\
& \leq C \bar{F}(x)\left(\mathrm{E}\left[L_{\vartheta 1}^{2}\left(T, m_{0}\right)\right]+\mathrm{E}\left[L_{\vartheta 2}^{2}\left(T, m_{0}\right)\right]\right)^{1 / 2} \mathrm{E}^{1 / 2}\left[L_{\vartheta 3}^{2}\left(T, m_{0}\right)\right] \\
& \leq C \bar{F}(x) \frac{\varepsilon}{m_{0}^{2 p_{2}+2}} \mathrm{E}^{1 / 2}\left[L_{\vartheta 3}^{2}\left(T, m_{0}\right)\right] .
\end{aligned}
$$

For $\mathrm{E}\left[L_{\vartheta 3}^{2}\left(T, m_{0}\right)\right]$, we derive, from (3.3),

$$
\mathrm{E}\left[L_{\vartheta 3}^{2}\left(T, m_{0}\right)\right] \leq C m_{0}^{2 p_{2}}\left(\left(m_{0}+1\right)^{2}+m_{0} \sum_{k=1}^{m_{0}} \mathrm{E}\left[\vartheta_{n}^{2 p_{2}}(T)\right]\right) \leq C m_{0}^{2 p_{2}+2}
$$


Combining the above formula with (3.16) we have

$$
I_{1}(T) \leq C \varepsilon \bar{F}(x) .
$$

Substituting (3.14) and (3.17) into (3.7), we obtain, for large $x$,

$$
\Psi(x, T) \leq C \varepsilon \bar{F}(x)+(1+\varepsilon) \sum_{n=1}^{\infty} \mathrm{P}\left\{X_{n} \vartheta_{n}(T)>x\right\} .
$$

On the other hand, we have

$$
\mathrm{E}\left[\vartheta_{1}^{p_{2}}(T)\right]=\mathrm{E}\left[\int_{0}^{T} \exp \left(-p_{2} L_{\theta}(t)\right) \lambda \mathrm{e}^{-\lambda t} \mathrm{~d} t\right]=\int_{0}^{T} \exp \left(t \psi_{\theta}\left(p_{2}\right)\right) \lambda \mathrm{e}^{-\lambda t} \mathrm{~d} t>0 .
$$

Hence, we have, $\mathrm{P}\left\{\vartheta_{1}(T)>1\right\}+C_{2}^{-1} \mathrm{E}\left[\vartheta_{1}^{p_{2}}(T) \mathbf{1}_{\left[\vartheta_{1}(T) \leq 1\right]}\right]>0$, where $C_{2}^{-1}$ is as defined in (2.1). Note that, for large enough $x$,

$$
\begin{aligned}
\mathrm{P}\left\{X_{1} \vartheta_{1}(T)>x\right\} & =\mathrm{P}\left\{X_{1} \vartheta_{1}(T)>x, \vartheta_{1}(T)>1\right\}+\mathrm{P}\left\{X_{1} \vartheta_{1}(T)>x, \vartheta_{1}(T) \leq 1\right\} \\
& \geq \mathrm{P}\left\{X_{1}>x, \vartheta_{1}(T)>1\right\}+C_{2}^{-1} \bar{F}(x) \mathrm{E}\left[\vartheta_{1}^{p_{2}}(T) \mathbf{1}_{\left[\vartheta_{1}(T) \leq 1\right]}\right] \\
& =\bar{F}(x)\left(\mathrm{P}\left\{\vartheta_{1}(T)>1\right\}+C_{2}^{-1} \mathrm{E}\left[\vartheta_{1}^{p_{2}}(T) \mathbf{1}_{\left[\vartheta_{1}(T) \leq 1\right]}\right]\right)
\end{aligned}
$$

By (3.18) and (3.19), we obtain, for large $x$,

$$
\begin{aligned}
\Psi(x, T) & \leq C \varepsilon \mathrm{P}\left\{X_{1} \vartheta_{1}(T)>x\right\}+(1+\varepsilon) \sum_{n=1}^{\infty} \mathrm{P}\left\{X_{n} \vartheta_{n}(T)>x\right\} \\
& \leq(1+C \varepsilon) \sum_{n=1}^{\infty} \mathrm{P}\left\{X_{n} \vartheta_{n}(T)>x\right\} .
\end{aligned}
$$

Next we consider the lower bound. By Lemma 3.2, (3.3), and (3.4), we find that, for any $\varepsilon>0$, there exists a positive integer $m_{0}=m_{0}(\varepsilon)$ such that, for any $m \geq m_{0}, p_{2}>\mathbb{J}_{F}^{+}$, and all large $x$,

$$
\begin{aligned}
\sum_{n=m}^{\infty} \mathrm{P}\left\{X_{n} \vartheta_{n}(T)>x\right\} & =\sum_{n=m}^{\infty} \mathrm{E}\left[\mathrm{P}\left\{X_{n} \vartheta_{n}(T)>x \mid \vartheta_{n}(T)\right\}\right] \\
& \leq \bar{F}(x) \sum_{n=m}^{\infty}\left(\mathrm{E}\left[\vartheta_{n}^{p_{2}}(T)\right]+\mathrm{P}\left\{\vartheta_{n}(T)<1\right\}\right) \\
& \leq C \varepsilon \bar{F}(x) .
\end{aligned}
$$

Since $\mathrm{E}\left[\int_{0}^{T} \exp \left(-L_{\theta}(t)\right) \mathrm{d} t\right]=\int_{0}^{T} \mathrm{E}\left[\exp \left(-L_{\theta}(t)\right)\right] \mathrm{d} t=\int_{0}^{T} \exp \left(t \psi_{\theta}(1)\right) \mathrm{d} t<\infty$, by we can take a positive number $A$ such that, for all $n=1, \ldots, m_{0}$,

$$
\mathrm{E}\left[\vartheta_{n}^{p_{2}}(T) \mathbf{1}_{\left[\int_{0}^{T} \exp \left(-L_{\theta}(t)\right) \mathrm{d} t>A\right]}\right]<\frac{\varepsilon}{m_{0}}, \quad \mathrm{P}\left\{\int_{0}^{T} \exp \left(-L_{\theta}(t)\right) \mathrm{d} t>A\right\}<\frac{\varepsilon}{m_{0} B^{p_{2}}},
$$

and let

$$
A_{2}=\left\{\bigcap_{1 \leq j \leq m_{0}}\left\{\eta \leq \vartheta_{j}(T) \leq B\right\}, \int_{0}^{T} \exp \left(-L_{\theta}(t)\right) \mathrm{d} t \leq A\right\}
$$


where $B$ and $\eta$ are defined in (3.8) and (3.9). Next we deal with the lower bound of $\Psi(x, T)$. By Lemma 3.4,

$$
\begin{aligned}
\Psi(x, T) \geq & \mathrm{P}\left\{\sum_{n=1}^{\infty} X_{n} \vartheta_{n}(T)>c \int_{0}^{T} \exp \left(-L_{\theta}(t)\right) \mathrm{d} t+x\right\} \\
\geq & \mathrm{P}\left\{\sum_{n=1}^{m_{0}} X_{n} \vartheta_{n}(T)>c A+x, A_{2}\right\} \\
= & \mathrm{E}\left[\mathbf{1}_{A_{2}} \mathrm{P}\left\{\sum_{n=1}^{m_{0}} X_{n} \vartheta_{n}(T)>c A+x \mid \vartheta_{k}(T), 1 \leq k \leq m_{0}, \int_{0}^{T} \exp \left(-L_{\theta}(t)\right) \mathrm{d} t\right\}\right] \\
\sim & \sum_{n=1}^{m_{0}} \mathrm{P}\left\{X_{n} \vartheta_{n}(T)>c A+x, A_{2}\right\} \\
\geq & \sum_{n=1}^{m_{0}} \mathrm{P}\left\{X_{n} \vartheta_{n}(T)>x+c A, \bigcap_{1 \leq j \leq m_{0}}\left\{\eta \leq \vartheta_{j}(T) \leq B\right\}\right\} \\
& -\sum_{n=1}^{m_{0}} \mathrm{P}\left\{X_{n} \vartheta_{n}(T)>x, \bigcap_{1 \leq j \leq m_{0}}\left\{\eta \leq \vartheta_{j}(T) \leq B\right\}, \int_{0}^{T} \exp \left(-L_{\theta}(t)\right) \mathrm{d} t>A\right\} \\
= & I_{12}(T)-I_{13}(T) .
\end{aligned}
$$

By the same approach used to prove $I_{4}(T)$ and $I_{5}(T)$, we obtain, for large $x$ and all $n=$ $1,2, \ldots, m_{0}$,

$$
\sum_{n=1}^{m_{0}}\left\{X_{n} \vartheta_{n}(T)>x, \bigcup_{1 \leq j \leq m_{0}}\left\{\eta>\vartheta_{j}(T)\right\}\right\} \leq C \varepsilon \bar{F}(x)
$$

and

$$
\sum_{n=1}^{m_{0}} \mathrm{P}\left\{X_{n} \vartheta_{n}(T)>x, \bigcup_{1 \leq j \leq m_{0}}\left\{\vartheta_{j}(T)>B\right\}\right\} \leq C \varepsilon \bar{F}(x)
$$

Note that $X_{n} \vartheta_{n}(T) \in \Im \cap \mathscr{D}$ by Lemma 3.1. Hence, for $I_{12}(T)$, by the property of $\& \cap \mathscr{D} \subset \mathcal{L}$ we have, for large enough $x>0$,

$$
\begin{aligned}
I_{12}(T) \geq & \sum_{n=1}^{m_{0}} \mathrm{P}\left\{X_{n} \vartheta_{n}(T)>x+c A\right\}-\sum_{n=1}^{m_{0}} \mathrm{P}\left\{X_{n} \vartheta_{n}(T)>x+c A, \bigcup_{1 \leq j \leq m_{0}}\left\{\eta>\vartheta_{j}(T)\right\}\right\} \\
& -\sum_{n=1}^{m_{0}} \mathrm{P}\left\{X_{n} \vartheta_{n}(T)>x+c A, \bigcup_{1 \leq j \leq m_{0}}^{\bigcup}\left\{\vartheta_{j}(T)>B\right\}\right\} \\
\geq & (1-\varepsilon) \sum_{n=1}^{m_{0}} \mathrm{P}\left\{X_{n} \vartheta_{n}(T)>x\right\}-C \varepsilon \bar{F}(x) .
\end{aligned}
$$


As for $I_{13}(T)$, by (2.1) we have, for large $x$,

$$
\begin{aligned}
I_{13}(T) & \leq \sum_{n=1}^{m_{0}} \mathrm{P}\left\{X_{n} B>x\right\} \mathrm{P}\left\{\int_{0}^{T} \exp \left(-L_{\theta}(t)\right) \mathrm{d} t>A\right\} \\
& \leq C_{2} m_{0} B^{p_{2}} \bar{F}(x) \mathrm{P}\left\{\int_{0}^{T} \exp \left(-L_{\theta}(t)\right) \mathrm{d} t>A\right\} \\
& \leq C \varepsilon \bar{F}(x) .
\end{aligned}
$$

Combining (3.20)-(3.23) and using (3.19), we obtain, for large $x$,

$$
\begin{aligned}
\Psi(x, T) & \geq(1-\varepsilon) \sum_{n=1}^{m_{0}} \mathrm{P}\left\{X_{n} \vartheta_{n}(T)>x\right\}-C \varepsilon \bar{F}(x) \\
& \geq(1-\varepsilon) \sum_{n=1}^{\infty} \mathrm{P}\left\{X_{n} \vartheta_{n}(T)>x\right\}-C \varepsilon \bar{F}(x) \\
& \geq(1-C \varepsilon) \sum_{n=1}^{\infty} \mathrm{P}\left\{X_{n} \vartheta_{n}(T)>x\right\} .
\end{aligned}
$$

where in the last step we have used (3.19).

\section{Acknowledgements}

Thanks are due to Professor Claudia Klüppelberg for helpful discussions. Dr Wang thanks Professor Joe Gani and Professor Ross Maller for their generous help. This work was supported by a MASCOS grant from the Australian Research Council. Wang's work was also supported by the Natural Science Foundation of China (grant number 70671018).

\section{References}

Bingham, N. H., Goldie, C. M. And Teugels, J. L. (1987). Regular Variation (Encyclopaedia Math. Appl. 27). Cambridge University Press.

Carmona, P., Petit, F. And Yor, M. (2001). Exponential functionals of Lévy processes. In Lévy Processes, eds O. E. Barndorff-Nielsen et al., Birkhäuser, Boston, MA, pp. 41-55.

Cline, D. B. H. And SAmorodnitsky, G. (1994). Subexponentiality of the product of independent random variables. Stoch. Process. Appl. 49, 75--98.

Cont, R. And Tankov, P. (2004). Financial Modelling with Jump Processes. Chapman and Hall/CRC, Boca Raton, FL.

Embrechts, P., KlüpPelberg, C. And Mikosch, T. (1997). Modelling Extremal Events (Appl. Math. 33). Springer, Berlin.

EMmer, S. AND KLÜPPELBERG, C. (2004). Optimal portfolios when stock prices follow an exponential Lévy process. Finance Stoch. 8, 17-44.

EMmer, S., KlÜPPELberg, C. AND Korn, R. (2001). Optimal portfolios with bounded capital at risk. Math. Finance 11, 365-384.

Frolova, A., Kabanov, Y. and Pergamenshchikov, S. (2002). In the insurance business risky investments are dangerous. Finance Stoch. 6, 227-235.

GAIER, J. AND GRANDITS, P. (2002). Ruin probabilities in the presence of regularly varying tails and optimal investment. Insurance Math. Econom. 30, 211-217.

Goldie, C. M. (1991). Implicit renewal theory and tails of solutions of random equations. Ann. Appl. Prob. 1, 126-166.

Hurst, S. R., Platen, E. and Rachev, S. T (1999). Option pricing for a logstable asset price model. Math. Comput. Modelling 29, 105-119.

Kalashnikov, V. AND Norberg, R. (2002). Power tailed ruin probabilities in the presence of risky investments. Stoch. Process. Appl. 98, 211-228. 
KLÜPPELBERG, C. AND KonstadinOVA, R. (2008). Integrated insurance risk models with exponential Lévy investment. Insurance Math. Econom. 42, 560-577.

Konstantinides, D. G. And Mikosch, T. (2005). Large deviations and ruin probabilities for solutions to stochastic recurrence equations with heavy-tailed innovations. Ann. Prob. 33, 1992-2035.

Korn, K. (1997). Optimal Portfolios. World Scientific, Singapore.

Kou, S. G. (2002). A jump-diffusion model for option pricing. Manag. Sci. 48, 1086-1101.

LiNDNeR, A. AND MALLER, R. (2005). Lev́y integrals and the stationarity of generalised Ornstein-Uhlenbeck processes. Stoch. Process. Appl. 115, 1701-1722.

Madan, D. B. And Seneta, E. (1990). The variance gamma (VG) model for share market returns. J. Business 63, 511-524.

PAulsen, J. (2002). On Cramér-like asymptotics for risk processes with stochastic return on investements. Ann. Appl. Prob. 12, 1247-1260.

Paulsen, J. and Gjessing, H. K. (1997). Ruin theory with stochastic return on investments. Adv. Appl. Prob. 29, 965-985.

Sato, K. I. (1999). Lévy Processes and Infinitely Divisible Distributions (Camb. Stud. Adv. Math. 68). Cambridge University Press.

TANG, Q. And Tsitsiashvili, G. (2003a). Precise estimates for the ruin probability in finite horizon in a discrete-time model with heavy-tailed insurance and financial risks. Stoch. Process. Appl. 108, 299-325.

TANG, Q. AND Tsitsiashvili, G. (2003b). Randomly weighted sums of subexponential random variables with application to ruin theory. Extremes 6, 171-188.

TANG, Q. AND Tsitsiashvili, G. (2004). Finite- and infinite-time ruin probabilities in the presence of stochastic returns on investments. Adv. Appl. Prob. 36, 1278-1299.

WANG, D. C. (2008). Finite-time ruin probability with heavy-tailed claims and constant interest rate. Stoch. Models 24, 41-57. 